\title{
Quality of uncomplicated malaria case management in Ghana among insured and uninsured patients
}

\author{
Ama P Fenny ${ }^{1,2^{*}}$, Kristian S Hansen ${ }^{3}$, Ulrika Enemark ${ }^{2}$ and Felix A Asante ${ }^{1}$
}

\begin{abstract}
Introduction: The National Health Insurance Act, 2003 (Act 650) established the National Health Insurance Scheme (NHIS) in Ghana with the aim of increasing access to health care and improving the quality of basic health care services for all citizens. The main objective is to assess the effect of health insurance on the quality of case management for patients with uncomplicated malaria, ascertaining any significant differences in treatment between insured and non-insured patients.
\end{abstract}

Method: A structured questionnaire was used to collect data from 523 respondents diagnosed with malaria and prescribed malaria drugs from public and private health facilities in 3 districts across Ghana's three ecological zones. Collected information included initial examinations performed on patients (temperature, weight, age, blood pressure and pulse); observations of malaria symptoms by trained staff, laboratory tests conducted and type of drugs prescribed. Insurance status of patients, age, gender, education level and occupation were asked in the interviews.

Results: Of the 523 patients interviewed, only 40 (8\%) were uninsured. Routine recording of the patients' age, weight, and temperature was high in all the facilities. In general, assessments needed to identify suspected malaria were low in all the facilities with hot body/fever and headache ranking the highest and convulsion ranking the lowest. Parasitological assessments in all the facilities were also very low. All patients interviewed were prescribed ACTs which is in adherence to the drug of choice for malaria treatment in Ghana. However, there were no significant differences in the quality of malaria treatment given to the uninsured and insured patients.

Conclusion: Adherence to the standard protocol of malaria treatment is low. This is especially the case for parasitological confirmation of all suspected malaria patients before treatment with an antimalarial as currently recommended for the effective management of malaria in the country. The results show that about 16 percent of total sample were parasitologically tested. Effective management of the disease demands proper diagnosis and treatment and therefore facilities need to be adequately supplied with RDTs or be equipped with well functioning laboratories to provide adequate testing.

Keywords: Quality of care, Uncomplicated malaria, Patient exit survey, Malaria treatment protocol, Ghana

\footnotetext{
*Correspondence: amafenny@yahoo.co.uk

${ }^{1}$ Economics Division, Institute of Statistical, Social and Economic Research

(ISSER), University of Ghana, P.O. Box LG 74, Legon LG74Accra, Ghana

${ }^{2}$ Department of Public Health, Aarhus University, Vennelyst Boulevard 6, 8000

Århus C, Denmark

Full list of author information is available at the end of the article
}

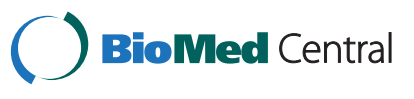

(c) 2014 Fenny et al.; licensee BioMed Central Ltd. This is an Open Access article distributed under the terms of the Creative Commons Attribution License (http://creativecommons.org/licenses/by/4.0), which permits unrestricted use, distribution, and reproduction in any medium, provided the original work is properly credited. The Creative Commons Public Domain Dedication waiver (http://creativecommons.org/publicdomain/zero/1.0/) applies to the data made available in this article, unless otherwise stated. 


\section{Introduction}

Malaria remains a leading cause of morbidity and mortality in sub-Saharan Africa. Malaria remains a leading cause of childhood illness and death in sub-Saharan Africa with an under five annual mortality of approximately a million [1]. It is the most significant public health problem in Ghana where it accounts for 38 percent of all outpatient illnesses, 35 percent of all admissions, and 34 percent of all deaths in children under five years [2]. Effective case management of uncomplicated malaria is a major strategy for malaria control [1]. This requires appropriate clinical assessment, laboratory proof of the disease either by microscopy or rapid diagnostic test (RDT) prior to treatment with an effective antimalarial [1]. Moreover, the Abuja declaration of May 2006, aims at achieving and sustaining universal access to appropriate interventions for all populations at risk of malaria. As a result, the goal of malaria control in Ghana is to reduce morbidity and mortality by 75 percent by 2015 [3].

Unfortunately, health services from both public and private providers are of questionable quality, with long waiting times, inaccurate diagnosis, inappropriate prescription and advice and frequent drug stock-outs. The use of presumptive malaria diagnosis without laboratory support is a common diagnostic procedure for malaria in Ghana and this predisposes patients to poor quality of malaria diagnosis and treatment [4]. A number of studies have shown that a large proportion of suspected malaria cases in Africa are given an antimalarial following presumptive diagnosis based on clinical symptoms only and without a parasitological test. This results in massive over-diagnosis of malaria, waste of antimalarial drugs and delay in appropriate treatments which in some cases have serious consequences [5-8].

The National Health Insurance Act, 2003 (Act 650) established the National Health Insurance Scheme (NHIS) with the aim of increasing access to health care and improving the quality of basic health care services for all citizens, especially the poor and vulnerable. The NHIS represents a major development in health system financing in Ghana. Since its introduction in 2005, levels of utilisation of health services has increased, as had been anticipated with the implementation of the scheme $[9,10]$. What was not anticipated clearly was the effect of this on infrastructure and staffing levels at health facilities. Some studies indicate that there has been limited improvement of facility infrastructure and staffing levels which has led to a strain on health workers [11-13]. It is unclear how the supply-side was strengthened to cope with these developments. There has been anecdotal information alleging that patients who are insured receive poor treatment at health facilities compared to non-insured. This could be due to the late reimbursement of claims by the National Health Insurance Authority (NHIA). Health facilities are more willing to serve the non-insured who make out-of pocket payments to the facilities for services rendered. The sustainability of the NHI scheme is in danger if members leave the scheme and further discourage others from enrolling.

To substantiate some of these anecdotal evidence, we needed to assess whether the insured and the non- insured are given the same standard of treatment when it comes to malaria care. First we assess the preparedness of facilities to manage the treatment of uncomplicated malaria. Secondly, we assess the adherence to malaria treatment guidelines noting any possible differences between the insured and uninsured groups. We hypothesise that both groups will receive the same treatment at the facility level. Finally we evaluate the association between patient satisfaction and quality of care received. Here, patient satisfaction relies on patients' perception of satisfaction on what was observed and how they were treated by providers. Uncomplicated Malaria is defined as the presence of fever or a recent history of fever, in the absence of any signs of severe disease [14].

Effective case management of malaria in the private and public health facilities will ultimately reduce malariarelated morbidity and mortality in Ghana. The information generated by this study will help design policy measures to strengthen the treatment component of the malaria control strategy and initiate improvement programmes.

\section{Background}

\section{Ghana's health system delivery and structure}

The Ministry of Health $(\mathrm{MOH})$ supervises and controls the policy formulation, monitoring and evaluation of activities and programmes geared towards achieving the targets set out in the health sector. The Ghana Health Service is responsible for delivery of public health and clinical services. Health care delivery is provided by both the public and private (private-for-profit and privatenot-for-profit) sectors, with the public sector organized according to national (teaching hospitals), regional (regional hospitals), district (district hospitals), sub-district (health centres) and Community-based Health Planning and Services (CHPS). At the sub-district level, health centres are the highest health facilities and first line of referral to the formal health services from the community clinic.

Beyond this setup, is an informal health care sector consisting of traditional practitioners who may be formally or informally trained. They include herbalists applying preparations from plant materials, naturopaths and homeopaths and spiritualists. Licensed and unlicensed druggists and traditional birth attendants (TBA) can also be found in this sector. Prior to the introduction of the NHIS, the user-fee regime encouraged many Ghanaians to selfmedicate, often relying on drug pedlars or self-prescription 
of medication [15]. Recent studies from Ghana and other developing countries where malaria is endemic show that drug shops are willing to sell cheap and less effective drugs; willing to sell less than the full course and nearly always rely on presumptive diagnosis [16-18].

\section{Overview of the NHIS}

The National Health Insurance Act, 2003 (Act 650) established the National Health Insurance Scheme (NHIS) with the aim of increasing access to health care and improving the quality of basic health care services for all citizens, especially the poor and vulnerable. The law establishing the scheme allows for the concurrently operation of DistrictWide (Public) Mutual Health Insurance schemes, Private Mutual Health Insurance schemes and Private Commercial Health Insurance schemes. The scheme covers inpatient hospital care, outpatient care at primary and secondary levels, and emergency and transfer services. The benefit package covers about $95 \%$ of treatment cases in Ghana including malaria, cervical and breast cancer, surgical operations, physiotherapy, maternity care, dental care and eye care. The NHIS scheme has an exemption policy to ensure that the poor and other vulnerable groups have access to healthcare. The exempt groups are children under the age of 18 years, the elderly above the age of 70 and the indigent (poor). A new NHIA law, Act 852 was established in November 2012 to make some adjustments to the previous law (Act 650). For instance, mental patients who were not adequately covered under the scheme have been included. The benefit package has also been extended to include any relevant family planning package to be provided under the National Health Insurance Scheme. As the need to balance access with quality of care is important to the success of the scheme, the new law enjoins the NHIA to collaborate with the relevant agencies to ensure quality healthcare to members of the Scheme and carry out clinical audits.

In order to become a member of the NHIS one needs to be registered and issued with a Health Insurance Membership ID Card. One needs to pay the appropriate premium (except those belonging to the exempt group) to benefit from the National Health Insurance Scheme. Children under the age of 18 and the elderly above 70 years must pay the registration fee which covers the administrative expenses for the issuance of their membership cards. The indigent are however exempted from both the registration fees and premiums. Membership is subject to yearly renewal and members must present their valid health insurance cards at the health facilities to benefit from the scheme. All public health facilities are automatically accredited to the NHIS. However, private health facilities have to apply to the National Health Insurance Authority (NHIA) for accreditation to participate in the scheme. Some of the accreditation criteria entail the number of qualified health personnel, availability and quality of utilities such as regular supply of water and electricity. Patients with valid NHIS cards may choose to access care either from public or private accredited health providers in the district.

\section{Defining and measuring quality of care}

The multi-faceted nature of healthcare and numerous stakeholders make quality of care a very difficult concept to define and measure [19-24]. To find a definition to capture all differing perceptions of what encompasses quality of care led the Institute of Medicine to define quality of care as "the degree to which health services for populations increase the likelihood of desired health outcomes and are consistent with current professional knowledge" [25]. In principle, quality is assured if patients can get the services they need and if the services provided are beneficial to them.

A number of frameworks have been developed in the past for quality of care assessment and many more have been formulated as variations of the former ones. They include the World Health Organisation (WHO) quality of care framework, the Bamako Initiative and more disaggregated approaches [19,26-29]. The disaggregated frameworks have gained popularity because they try and capture the complexity and multi-dimensionality of quality of care [28]. This study adopts the Donabedian Model which has been widely used in the healthcare quality field and has been applied across a spectrum of medical specialties and illness diagnoses $[19,30]$.

The framework divides factors impacting quality into structures, processes and outcomes, connected by unidirectional arrows in that order. Structure includes all the factors that affect the context for health care delivery. The physical facility, equipment, human resources, as well as organisational characteristics such as supervision are some of the areas classified under structure. Structure is often easier to observe and measure. Process refers to the interaction between providers and their patients and relates to how provider tasks and clinical processes are both organized and performed. It also includes providers' ability to communicate and build trust with patients [29]. Donabedian defines this as interpersonal care and it includes "the management of the social and psychological interaction between client and practitioner" [19]. Outcomes include the effects of healthcare on patients or populations, changes to health status, health behaviour, patient satisfaction and health-related quality of life [30]. However, accurately measuring outcomes that can be attributed exclusively to healthcare is very difficult [19].

Donabedian's model is however criticised for its sequential and linear progression from structure to process and outcome. The model suggests a directional link from the structure to processes of care and finally to patient 
outcomes [31]. Some suggest that there are other important factors such as patient characteristics and environmental factors that need to be incorporated for a more complete evaluation of quality care [31-33]. The model however, allows researchers to draw conceptual models that are suitable to their own health systems incorporating in them all the aspects that are needed to holistically evaluate their health interventions [34]. On the other hand, examining the process of care itself rather than its outcomes also provides good information on how medical care is being applied. For instance, the examination of processes of care such as clinical history, physical examination and diagnostic tests; technical ability to perform diagnostic procedures, continuity of care and acceptability of care to the patient provide relevant information that could guide the process of improving outcomes.

\section{Conceptual framework}

Based on the Donabedian framework we construct a model to demonstrate the various links from structure to outcome (Figure 1). Structure of care includes amenities, equipment, drug supplies, utilities (water and power supply) and trained personnel. We define the process of care in terms of technical quality (adherence to clinical guidelines) and interpersonal aspects of care (attitude of staff towards clients). Measurement of process is often preferred because process is under relatively greater control of providers, needs a shorter time frame for results and can directly inform improvement [35]. Finally, we define patient satisfaction as an intermediate outcome which should lead to the ultimate outcome of health improvement.

\section{Malaria treatment protocol in Ghana}

As part of process quality, the study focuses on the adherence to malaria treatment guidelines for uncomplicated malaria in Ghana. The standard for assessing the quality of malaria testing, diagnosis, and treatment in Ghana is presented in Figure 2. The flow chart presents all the different scenarios and steps to be taken in the treatment process. Uncomplicated malaria is mainly clinically diagnosed based on fever as case definition. In health facilities, the current approach is to confirm the clinical diagnosis with confirmation by parasitological test; either Rapid Diagnostic Test (RDT) or microscopy [14]. Currently, the use of RDTs is recommended for the diagnosis of malaria in lower level peripheral facilities (CHPS), but not in health centres and hospitals where microscopy is recommended. Microscopic testing should be the standard at health facilities, including district hospitals and higher level facilities. Laboratory services exist at most levels of health facility, except smaller health centres and therefore we expect facilities to be equipped to carry out the necessary diagnostic tests.

As noted in the standard protocol, in the initial assessment, the patient suffering from uncomplicated malaria commonly complains of: fever or a history of fever within the preceding 2-3 days, chills (feeling unusually cold),

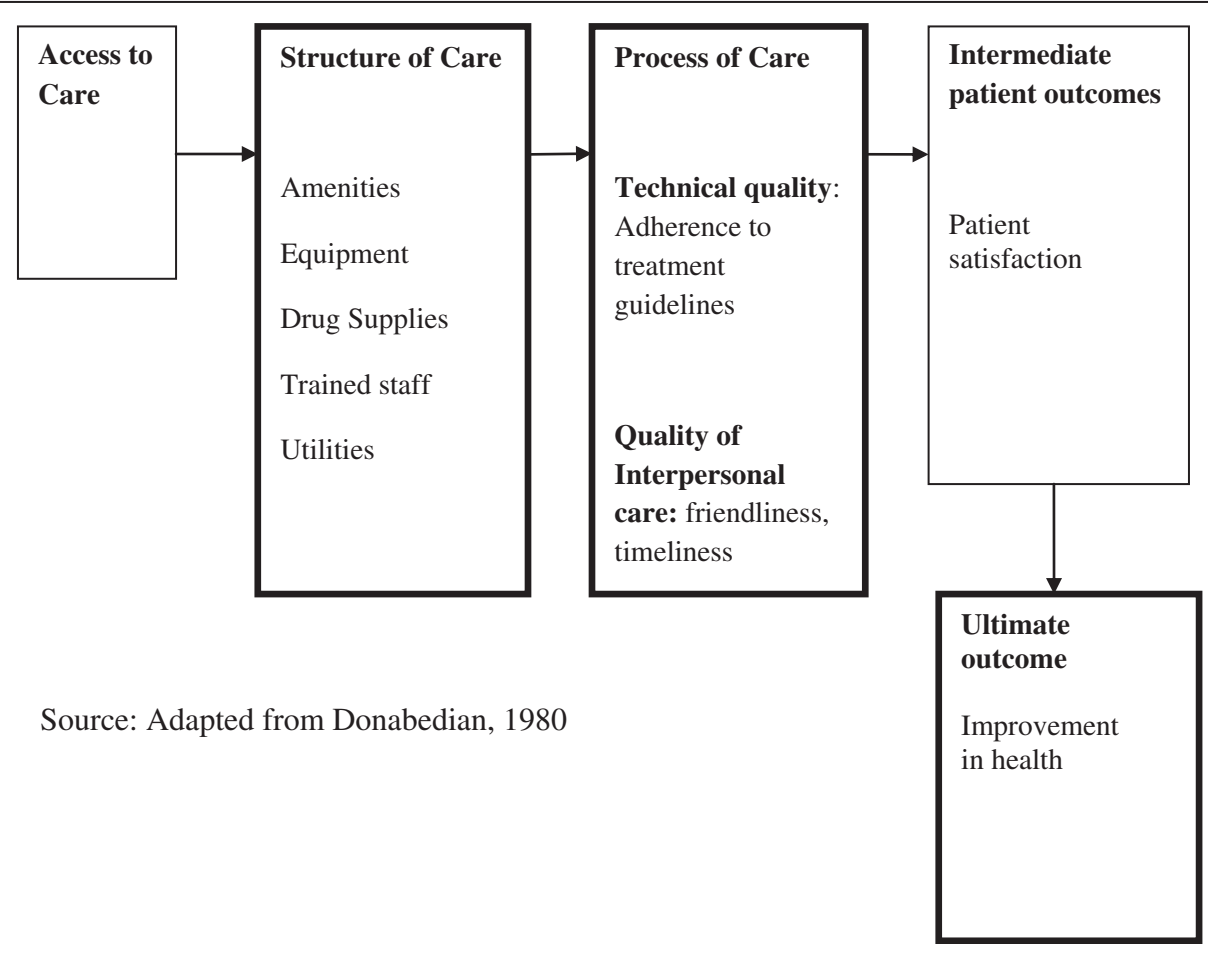

Figure 1 Donabedian's structure-process-outcome paradigm. 


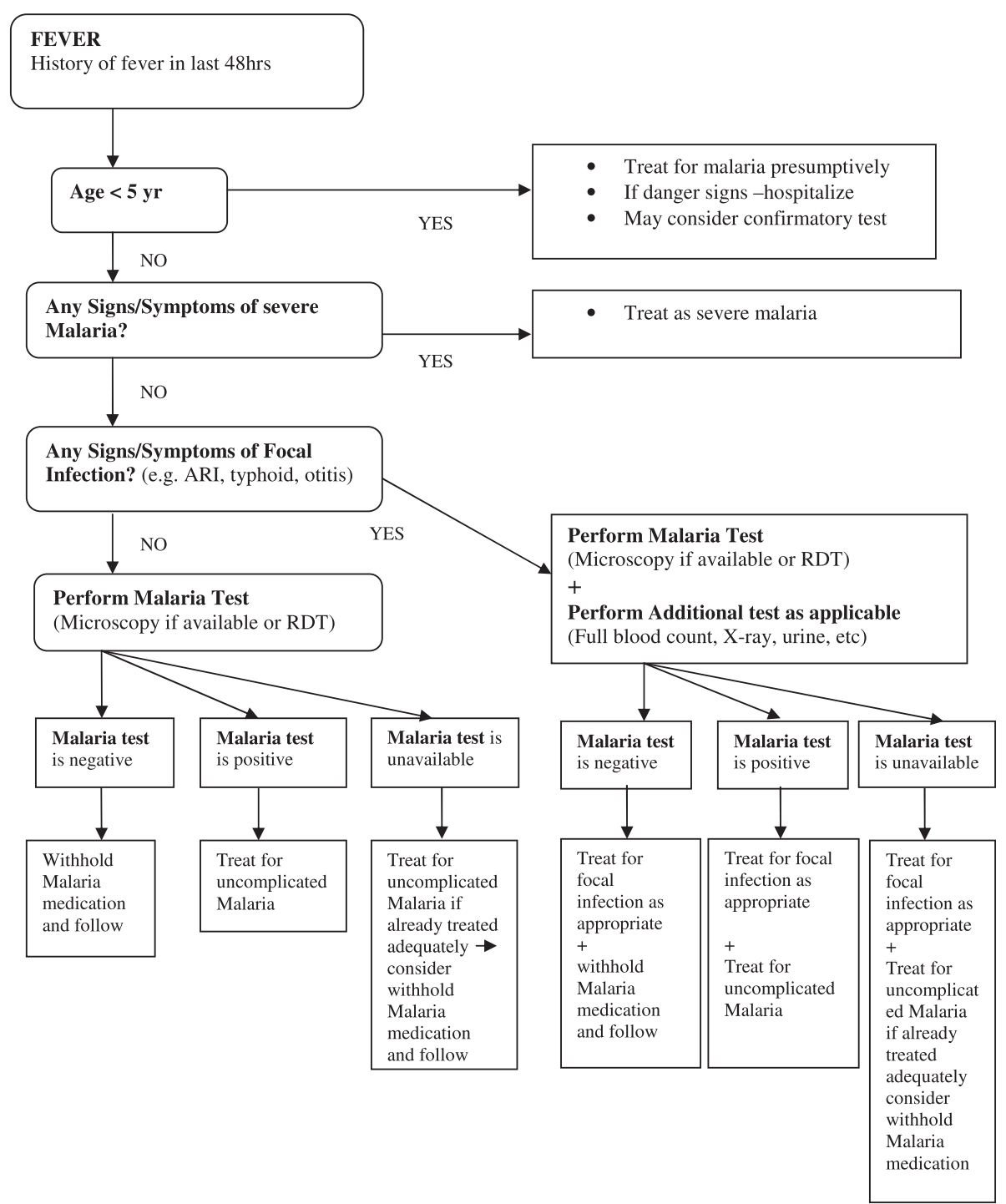

Source: MOH (2009) Training Manual for the Management of Malaria at Health Facilities in Ghana

Figure 2 Flow chart for the diagnosis and treatment of uncomplicated malaria.

rigors (shivering) and headache. Other clinical features may include generalised body and joint pain, nausea and or vomiting, loss of appetite, sweating, abdominal pain (especially in children), bitterness in the mouth, irritability and refusal to feed (in infants). These features may occur one at a time or occur in combination. As part of the protocol a complete history is required from each patient and any presenting symptoms are noted. In patients with a suspected case of uncomplicated malaria, a parasitological confirmation is recommended, wherever possible, before giving antimalarial treatment (Figure 2).

The WHO Guidelines recommend a parasitological confirmation of diagnosis in all patients suspected of having malaria before treating [1]. The standard protocol for Ghana currently specifies that under-5 s with a febrile illness should be treated for malaria without testing (Figure 2). Other issues clarified include how patients should be managed if malaria testing is not available. However, in line with WHO guidelines the protocol highlights the importance of performing malaria tests and other tests where necessary before malaria medication is supplied to patients with the disease. Artesunate-Amodiaquine Combination is the combination drug of choice for the treatment of uncomplicated malaria as a first line treatment. The second line treatment is the recommended strengths and dosage forms of Artemether-Lumefantrine or Dihydroartemisinin Piperaquine [14]. 


\section{Study objectives}

Based on the Donabedian framework, there are three main objectives in this study. The first is to assess the structural quality by considering the preparedness of facilities to manage the treatment of uncomplicated malaria. Process quality is assessed in two ways; technical quality where the adherence to malaria treatment guidelines is assessed and interpersonal quality which considers the attitude of personnel towards patients. The outcome assesses patient satisfaction with the overall quality of care received at the health facility.

\section{Methods}

\section{Study design}

The 10 administrative regions in Ghana are subdivided into 170 districts which cut across 3 agro-ecological zones in Ghana namely coastal, forest and savannah. For this study a district was selected in each zone making a total of 3 districts surveyed. The study was a crosssectional survey consisting of provider interviews at selected facilities in the sample, an audit of staffing, equipment and procedures routinely conducted in antimalarial treatment prescribed to fever cases as well as exit interviews with clients through structured questionnaires.

Malaria is hyperendemic in all parts of the country, with all the 22.4 million population at risk. Transmission occurs all year round with slight seasonal variations during the rainy season from April to July [14]. The main parasite species causing malaria in Ghana are Plasmodium falciparum (80-90\%), Plasmodium malariae (20-36\%), and Plasmodium ovale $(0.15 \%)$. Mixed infections of P. falciparum and P. malariae are not uncommon [14].

\section{Provider interview}

An initial mapping of the various types of health facilities provided in the district was conducted. The sample size was determined by considerations of the range of providers and feasibility. The sampling frame included the major types of private and public providers in each district. Public providers were district hospitals; health centres (HCs) and CHPS. All private providers in this study are accredited by the National Health Insurance Authority (NHIA) meaning that members with valid NHIS cards are able to benefit from approved services with no charge at the point use. The study sites for each district selected included the district hospital, 3 randomly selected health centres and 3 randomly selected community health posts (CHPS). The survey took place between January and March, 2011.

A structured questionnaire was administered to the heads or owners of selected public and private providers. The quality of antimalarial treatment prescribed to fever cases elicited from the various providers included service availability, human resource availability, and adherence to treatment standards and protocols (method of diagnosis of malaria; laboratory capacity and provision of ACTs and other anti-malarial drugs). This was based on the premise that appropriate treatment consists of proper diagnosis and treatment with nationally recommended drugs based on the guidelines. Other variables that were explored included assets (electricity, water, equipment) of the facilities and the general cleanliness of the environment.

\section{Exit interviews}

\section{Sampling for Exit interviews}

The sample size for each district was 200 patients expected to be distributed equally between the insured and uninsured. Patients who reported being diagnosed with uncomplicated malaria and prescribed antimalarials were interviewed. The number of patients to be interviewed per selected health facility was obtained based on the daily average number of malaria cases presented in the health facilities. A random systematic sampling approach was used to select patient for interviews over the study period in each district. To start, the first patient was randomly chosen (e.g. the fourth patient who met with the criteria for selection). Subsequently, every second patient following the initially selected patient was sampled. Approximately $3-10$ patients were interviewed a day depending on which type of facility (district hospital, health centre or CHPS) was surveyed. Data was collected on initial examinations performed (temperature, weight, age, blood pressure and pulse); observations of malaria symptoms by trained staff, laboratory tests conducted and types of drug prescribed. Insurance status of patients (whether patient was a valid cardholder), age, gender, education level and occupation were asked in the interviews.

\section{Ethical approval and consent}

Ethical clearance was given by Institutional Review Board (IRB), of the Noguchi Memorial Institute for Medical Research (NMIMR). Informed consent was obtained from the respondent who read and agreed to be interviewed having accepted the conditions.

\section{Data analysis}

Tabulations were used to examine variables for stated quality elicited from the various providers in the different areas. Tabulations using Chi-square test to examine adherence to treatment standards and protocols and differences between the insured and uninsured patients. This was based on the premise that appropriate treatment consists of proper diagnosis and treatment. These variables were the clients' responses to whether specific processes of care were undertaken by trained personnel. Tabulations were also used to examine patient satisfaction with quality of care at the health facilities. 


\section{Results}

Patient exit survey - demographic and background statistics

Table 1 presents detailed findings of the demographic data and background characteristics. Our sample consisted of a total of 523 patients who were given treatment for malaria at the various facilities in all 3 districts. Out of the total sample 92 percent were insured and 8 percent non-insured; 64 percent males and 36 percent females. Patients under 5 years old accounted for 33 percent of the total sample with those above 70 years accounting for 4 percent. District hospitals accounted for the highest number followed by private hospitals and then health centres followed by the CHPS because of the sampling procedure. Out of the 40 uninsured patients only 3 sought care at private hospitals (Figure 3 ).

\section{Structure quality of care}

\section{General characteristics of the providers}

Four main providers were assessed for the study: district hospitals, private hospitals, health centres and CHPS.

Table 1 Demographic and background statistics

\begin{tabular}{|c|c|c|}
\hline Variables & Frequency & $\%$ \\
\hline \multicolumn{3}{|l|}{ Sex } \\
\hline Female & 186 & 35.56 \\
\hline Male & 337 & 64.44 \\
\hline \multicolumn{3}{|l|}{ Age } \\
\hline$<5$ & 171 & 32.70 \\
\hline 5-17 years & 96 & 18.36 \\
\hline $18-60$ years & 235 & 44.93 \\
\hline$\geq 70$ years & 21 & 4.02 \\
\hline \multicolumn{3}{|l|}{ Education } \\
\hline No education & 132 & 39.76 \\
\hline Some primary & 104 & 31.33 \\
\hline JSS/Middle & 85 & 25.60 \\
\hline Secondary or higher & 11 & 3.31 \\
\hline \multicolumn{3}{|l|}{ Facility type } \\
\hline CHPS & 12 & 2.29 \\
\hline Public health centres & 98 & 18.73 \\
\hline Private hospitals & 131 & 25.05 \\
\hline District hospitals & 282 & 53.92 \\
\hline \multicolumn{3}{|l|}{ Insurance status } \\
\hline Insured & 483 & 92.35 \\
\hline Uninsured & 40 & 7.65 \\
\hline Nearest facility to patient & 319 & 60.99 \\
\hline \multicolumn{3}{|l|}{ Yes } \\
\hline No & 204 & 39.01 \\
\hline $\mathbf{N}$ & 523 & \\
\hline
\end{tabular}

Source: Patient exit data, January to April, 2011.
All the respondents of the survey were heads of the facilities visited. All the facilities had trained personnel who were appropriate for each type of facility. All the health centres and CHPS were manned by trained medical assistants or midwives. There were doctors available in all the hospitals visited.

\section{Availability of functional equipment}

All the facilities had functioning thermometers, weighing scales and cold boxes/fridges (Table 2). Essential malaria drugs including ACTs were available at the time of the survey with the exception of the CHPS compound where the facility was not equipped to handle severe malaria cases and therefore lacked quinine and intravenous (IV) fluid (Table 2).

\section{Laboratory capacity}

All the facilities had trained personnel on how to use RDTs if there were no laboratories available. Four out of the 7 public health centres (57\%) had no medical laboratories and therefore referred severe malaria cases to the district hospital. Only 2 out of the 7 health centres had RDTs in stock and therefore could offer the RDT to patients (Table 2). The rest had run out of stock and were still waiting for supplies from the Central Medical Stores (CMS).

\section{Process quality of care Quality of clinical assessment and use of diagnostic testing} The vital statistics are often taken by nurses or health care assistants prior to consultation with trained personnel (doctors, nurses, midwives or medical assistants depending on which type of facility is visited). Routinely, the client's age, weight, temperature, pulse and blood pressure are recorded. The results show that in all the different types of facility a high proportion of clients had their temperatures taken and weight recorded (Table 3). The least recorded vital statistics was the pulse. There were significant differences between the insured and uninsured groups with the exception of taking of blood pressure and pulse.

The quality of clinical assessments was evaluated by measuring the proportion of patients for whom health workers had ascertained if a given sign or symptom was present. This could be elicited from the response given to a question asked or provided by the client spontaneously during consultation. In general, assessments needed to identify suspected malaria were low in all the facilities with convulsion ranking the lowest. There were no significant differences between the two groups for most of the symptoms checked except with loss of appetite and yellowish urine (Table 4).

Parasitological assessment in all the facilities was very low with 4 percent of the insured and 10 percent of the 


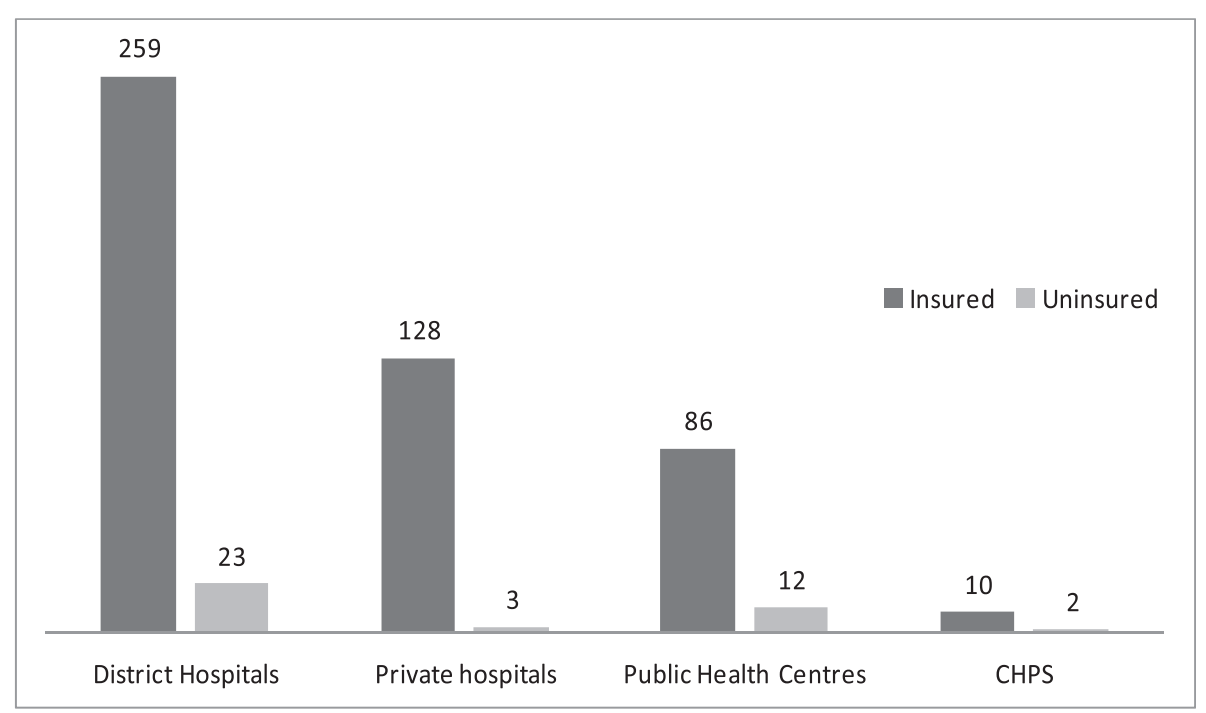

Source: Patient exit data, January to April, 2011

Figure 3 Distribution of outpatients interviewed by type of health care providers and insurance status.

uninsured tested using RDTs. For laboratory testing, 15 percent of the insured were tested for malaria compared to 25 percent of the uninsured (Table 5).

\section{Outcome quality of care}

\section{Patient satisfaction}

In total, 93 percent were satisfied with overall care and $7 \%$ were not satisfied. Similar trend was reported in the insured and uninsured groups. Satisfaction levels were highest in the health centres where 98 percent of those attending the facilities were satisfied with overall care (Table 6). There were no clear differences between the two groups in relation to the quality of care variables with the exception of waiting time and satisfaction at the pharmacy/dispensary (Table 7). Proportionally, more of the uninsured patients were satisfied with the waiting

Table 2 Availability of equipment, and medicines needed to manage malaria in outpatient health facilities

\begin{tabular}{|c|c|c|c|c|}
\hline & $\begin{array}{l}\text { District Hospital } \\
\mathrm{N}=3\end{array}$ & $\begin{array}{l}\text { Private hospital } \\
\mathrm{N}=1\end{array}$ & $\begin{array}{l}\text { Public health centres } \\
\mathrm{N}=7\end{array}$ & $\begin{array}{l}\text { CHPS } \\
\mathrm{N}=1\end{array}$ \\
\hline \multicolumn{5}{|l|}{ Characteristics } \\
\hline Thermometer & 3 & 1 & 7 & 1 \\
\hline Functional scale for weighing & 3 & 1 & 7 & 1 \\
\hline Cold box/fridge & 3 & 1 & 7 & 1 \\
\hline \multicolumn{5}{|l|}{ Laboratory capacity } \\
\hline Medical laboratory & 3 & 1 & 4 & 0 \\
\hline Staff trained to perform microscopy & 3 & 1 & 4 & 0 \\
\hline Functional microscope, according to the laboratory technician & 3 & 1 & 4 & 0 \\
\hline Staff trained to perform RDTs & 3 & 1 & 7 & 1 \\
\hline Malaria testing, by microscopy & 3 & 1 & 4 & 0 \\
\hline Malaria testing, by both microscopy and RDT & 2 & 1 & 2 & 1 \\
\hline \multicolumn{5}{|l|}{ Medicines in stock } \\
\hline ACTs & 3 & 1 & 6 & 1 \\
\hline Quinine & 3 & 1 & 6 & 0 \\
\hline I.M. Artemeter & 3 & 1 & 5 & 1 \\
\hline IV -Fluid & 3 & 1 & 6 & 0 \\
\hline Artemisinin-based suppository & 2 & 1 & 4 & 1 \\
\hline
\end{tabular}

Source: Patient exit data January to April, 2011. 
Table 3 Vital statistics observed by different health care providers

\begin{tabular}{lll}
\hline Vital statistics & $\begin{array}{l}\text { Insured } \\
\mathbf{N}=\mathbf{4 8 3}(\%)\end{array}$ & $\begin{array}{l}\text { Uninsured } \\
\mathbf{N}=\mathbf{4 0}(\%)\end{array}$ \\
\hline Temperature & $78^{* *}$ & $97^{* *}$ \\
Age & $49^{* *}$ & $80^{* *}$ \\
Weight & $95^{*}$ & $88^{*}$ \\
Blood pressure & 53 & 43 \\
Pulse & 27 & 38 \\
\hline
\end{tabular}

p- Chi-square test.

**Significant at $1 \%$.

*Significant at 5\%.

Source: Patient exit data January to April, 2011.

time and the pharmacy/dispensary compared to the insured $(73 \%$ vs. $62 \%)$. The perception of quality of care received at a facility influences decisions as to whether to return and whether to recommend the service to other potential users (family members or friends). From the patient exit survey, about 98 percent of the total number would return to the health facility and will also recommend the facility to friends and family. This was the same in both the uninsured and insured groups (Table 8 ).

\section{Discussion}

This study sets out to ascertain the significant differences between the quality of structure, process and outcome of care for antimalarial treatment prescribed to

Table 4 Malaria symptoms that the insured and uninsured patients were asked about by providers

\begin{tabular}{lll}
\hline Malaria symptoms & Insured & Uninsured \\
\hline Hot body/fever & 82 & $\mathbf{N}=\mathbf{4 0}$ (\%) \\
Weakness & 53 & 88 \\
Cough & 62 & 58 \\
Vomiting & 58 & 55 \\
Convulsion & 6 & 65 \\
Joint pains & 35 & 5 \\
Nausea & 21 & 30 \\
Chills and shivering & 41 & 23 \\
Yellowish eye ball & 40 & 48 \\
Catarrh & 21 & 35 \\
Diarrhoea & 50 & 25 \\
Headache & 58 & 50 \\
Loss of appetite & $36^{*}$ & 58 \\
Bitter taste & 23 & $53^{*}$ \\
Yellowish urine & & 28 \\
\hline *- Chi-square test. & Significant at 5\%. & \\
Source: Patient exit data January to & 2011. & \\
\hline & & \\
\hline
\end{tabular}

Table 5 Parasitological test performed

\begin{tabular}{lll}
\hline Laboratory assessment & Insured & Uninsured \\
(RDT or microscopy ) & $\mathbf{N}=\mathbf{4 8 3}(\%)$ & $\mathbf{N = 4 0}(\%)$ \\
\hline Yes & 19 & 35
\end{tabular}

Source: Patient exit data January to April, 2011.

fever cases among insured and uninsured patients compared to the standard protocol outlined in the guidelines for the treatment of malaria. A limitation of the present study is that the low number of uninsured in the exit interview did not allow for a more rigorous assessment of possible differences between the insured and uninsured. The GHS 2011 annual report shows that the proportion of OPD attendance by insured clients increased from 55.8 percent in 2010 to 82.1 percent in 2011 [2]. It was assumed at the start of the study that there may be almost equal numbers of the insured as uninsured. Notwithstanding this, the information shows what truly exists on the ground. The fact that majority of respondents were card holders confirms the results of previous studies $[9,10]$.

Going by Donabedian's 'structure-process-outcome' model, we identify a number of shortfalls with case management of malaria among outpatients with uncomplicated malaria. First is the use of presumptive malaria diagnosis without laboratory support, which seems to be a common diagnostic procedure in both public and private facilities surveyed. Although majority observed vital statistics, very few of the symptoms of malaria were checked for. In addition, less than 15 percent of the patients in total were diagnosed and treated for uncomplicated malaria in the public and private health facilities based on test results from microscopy or RDTs. Presumptive treatment for malaria seems to be widely practised across all levels of healthcare provision with the exception of CHPS where RDTs were available and used on all occasions.

Table 6 Number of patients (\%) who were satisfied with overall care, patient exit survey

\begin{tabular}{llll}
\hline & $\mathbf{N}$ & Satisfied (\%) & Not satisfied (\%) \\
\hline Total & 523 & 93.3 & 6.7 \\
Insurance status & & & \\
Insured & 483 & 93.6 & 6.4 \\
Uninsured & 40 & 90 & 10 \\
$p=0.384 x^{2}=0.759$ & & & \\
Facility type & & & 7.8 \\
District Hospital & 282 & 92.2 & 8.4 \\
Private hospital/clinic & 131 & 91.6 & 1.8 \\
Health centres/CHPS & 110 & 98.2 & \\
$p=0.069 x^{2}=5.350$ & & & \\
\hline
\end{tabular}

Source: Patient exit data January to April, 2011. 
Table 7 Number of patients (\%) who were satisfied with care by quality of care dimensions and health insurance status

\begin{tabular}{|c|c|c|c|}
\hline Quality of care variables & $\mathbf{N}$ & Satisfied (\%) & Not satisfied (\%) \\
\hline \multicolumn{4}{|l|}{ Waiting time } \\
\hline Insured & 483 & 61.5 & 38.5 \\
\hline Uninsured & 40 & 72.5 & 27.5 \\
\hline \multicolumn{4}{|l|}{$p=0.167 x^{2}=1.907$} \\
\hline \multicolumn{4}{|l|}{ Friendliness of staff } \\
\hline Insured & 483 & 97.5 & 2.5 \\
\hline Uninsured & 40 & 97.5 & 2.5 \\
\hline \multicolumn{4}{|l|}{$p=0.849 x^{2}=0.327$} \\
\hline \multicolumn{4}{|l|}{ Satisfaction at reception } \\
\hline Insured & 483 & 55.8 & 44.2 \\
\hline Uninsured & 40 & 57.5 & 42.5 \\
\hline \multicolumn{4}{|l|}{$p=0.836 x^{2}=0.043$} \\
\hline \multicolumn{4}{|c|}{ Satisfaction with consultation } \\
\hline Insured & 483 & 97.9 & 2.1 \\
\hline Uninsured & 40 & 97.5 & 2.5 \\
\hline \multicolumn{4}{|l|}{$p=0.856 x^{2}=0.033$} \\
\hline \multicolumn{4}{|c|}{ Satisfaction at pharmacy/dispensary } \\
\hline Insured & 483 & 64.6 & 35.4 \\
\hline Uninsured & 40 & 72.5 & 27.5 \\
\hline$p=0.313 x^{2}=1.017$ & & & \\
\hline
\end{tabular}

Table 8 Number of patients (\%) who received all medicines, were asked for follow-up, will revisit facility and recommend facility to friends/relatives

\begin{tabular}{llll}
\hline & N & Yes (\%) & No (\%) \\
\hline Received all prescribed medicines & & & \\
Insured & 483 & 94.2 & 5.8 \\
Uninsured & 40 & 95 & 5 \\
$p=0.835 X^{2}=0.043$ & & & \\
Follow-up review requested & & & \\
Insured & 483 & 43.1 & 56.9 \\
Uninsured & 40 & 45 & 55 \\
$p=0.812 X^{2}=0.056$ & & & \\
Will revisit facility & & & \\
Insured & 483 & 97.5 & 2.5 \\
Uninsured & 40 & 87.5 & 12.5 \\
$p=0.001 X^{2}=11.783$ & & & \\
Will recommend facility to friends/relatives & & & \\
Insured & 483 & 97.7 & 2.3 \\
Uninsured & 40 & 87.5 & 12.5 \\
$p=0.000 X^{2}=13.017$ & & \\
\hline
\end{tabular}

Source: Patient exit data January to April, 2011.
The standard protocol highlights the need for systematic laboratory testing but clearly this has not been adhered to in the health facilities. This phenomenon is not restricted to Ghana; other African countries where malaria is endemic report the very low rates of the use of malaria diagnostics. Dodoo et al. in a study from 2007 to show the mode of diagnosis and pattern of drug management in outpatients diagnosed with suspected uncomplicated malaria, indicate that only 3.2 percent of total diagnoses were parasitologically diagnosed [36]. Nationally, there has been reported improvement in the percentage of OPD malaria cases tested, from 14 percent in 2009 to 18.9 percent in 2011 [2]. However, the problem is even more entrenched in cases where providers have been known to have prescribed malaria drugs when microscopy cases have been shown to be negative [5,6,8,37].

The study shows that although, all the structure elements are often available it doesn't guarantee that the process elements will be followed through. For instance all the district hospitals and the private hospital in the sample had medical laboratories with functional microscopes and trained staff to perform malaria testing by microscopy yet very few of them parasitologically diagnose the malaria cases. There is clearly an adequate provision of essential diagnostic services (microscopy or RDTs) for malaria diagnosis in the health 
facilities but little use and this suggests very weak monitoring of the quality of malaria diagnostics.

On the outcomes, patient satisfaction ratings were high with many of them willing to revisit and recommend the facilities to friends and families. There was significantly no difference between the insured and uninsured patients. However, more of the insured patients were dissatisfied with the waiting time and the services offered at the pharmacies/dispensaries. We are limited in making any conclusive judgments because of the very small numbers of the uninsured. Yet the results of a similar study in two districts in the northern Ghana indicate that the long delay at pharmacies was one of the major complaints by insured patients [38].

\section{Conclusion}

Compared to the national malaria protocol for the treatment of malaria and the current recommendation by the WHO on parasitological testing of all febrile cases, this study has revealed a clear short coming in the process care of the treatment of uncomplicated malaria in the selected facilities. The quality in terms of undertaking of routine microscopy was poor in all health facilities, regardless of their level. Some public health centres had no laboratories at all although this is vital to the performance of malaria diagnostic tests in the country. Treating all fevers presumptively as malaria can hide underlying fatal conditions. We recommend that these facilities are equipped with well functioning laboratories to provide adequate testing. It is also recommended to improve the capacity for RDT use in facilities where laboratories have not been provided with technical assistance provided.

The fact that very few uninsured patients presented with uncomplicated malaria cases limits our comparative analysis of antimalarial treatment prescribed to fever cases between the two groups. However, the very few numbers of uninsured raises red flags, as we have no a priori reason to believe that the uninsured suffer less from malaria than the insured. We are not sure where the uninsured are seeking care. Treatment interventions meant to reduce malaria mortality or morbidity is most often facility-based and may therefore be missed by the ill who seek care outside of the health facilities. Patient satisfaction levels were quite high whether by insurance or facility type but more information about quality differentials across providers is needed to identify the key areas for intervention. The study design was such that we may have missed a number of dissatisfied patients who no longer used the facilities and therefore the patient satisfaction ratings may have been slightly exaggerated. We recommend further studies to allow for more analysis on the quality of healthcare services for antimalarial treatment prescribed to fever cases.

\section{Competing interests}

The authors declare that they have no competing interests.

\section{Authors' contributions}

APF was involved in the study design, training of research assistants, review of data collection tools, data collection, data entry, data analysis, drafting the manuscript, revising and writing of the final manuscript. KSH, UE and FAA contributed to the design, review of data collection tools, data analysis, critical review of the manuscript and revising the manuscript. All authors read and approved the final manuscript.

\section{Author details}

${ }^{1}$ Economics Division, Institute of Statistical, Social and Economic Research (ISSER), University of Ghana, P.O. Box LG 74, Legon LG74Accra, Ghana.

2Department of Public Health, Aarhus University, Vennelyst Boulevard 6, 8000 Århus C, Denmark. 'Department of Global Health and Development, Faculty of Public Health and Policy, London School of Hygiene and Tropical Medicine, 15-17 Tavistock Place, WC1H 9SH London, UK.

Received: 21 February 2014 Accepted: 4 July 2014

Published: 24 July 2014

\section{References}

1. World Health Organization: Roll Back Malaria Partnership: Global Malaria Action Plan. For a Malaria-Free World. Geneva World Health Organisation (2010) Guidelines for the Treatment of Malaria. 2nd edition. Geneve: WHO Press; 2010.

2. Ghana Health Service: Annual Report, (2011). Accra: Ghana Health Service; 2012.

3. Ghana Health Service/Ministry of Health: Ghana Malaria Strategic Plan: 2008-2015. Accra, Ghana: Ghana Health Service/Ministry of Health; 2007. http://www.ghanahealthservice.org/includes/upload/publications/STRATEGIC \%20PLAN.pdf. Accessed 12-07-2014.

4. USAID/CDC/QHP, GHS/NMCP, WHO: Ghana - Nationwide Malaria Health Facility Survey. Accra, Ghana: Quality Health Partners; 2008.

5. Reyburn H, Ruanda J, Mwerinde O, Drakeley C: The contribution of microscopy to targeting antimalarial treatment in a low transmission area of Tanzania. Malar J 2006, 5:4. http://dx.doi.org/10.1186/1475-2875-5-4.

6. Hamer DH, Ndhlovu M, Zurovac D, Fox M, Yeboah-Antwi K, Chanda P, Sipilinyambe N, Simon JL, Snow RW: Improved diagnostic testing and malaria treatment practices in Zambia. JAMA 2007, 297:2227-2231. http://dx.doi.org/10.1001/jama.297.20.2227.

7. Rowe AK, Ponce de León GF, Mihigo J, Santelli AC, Miller NP, Van-Dúnem P: Quality of malaria case management at outpatient health facilities in Angola. Malar J 2009, 8:275. http://dx.doi.org/10.1186/1475-2875-8-275.

8. Ansah EK, Narh-Bana S, Epokor M, Akanpigbiam S, Quartey AA, Gyapong J, Whitty CJ: Rapid testing for malaria in settings where microscopy is available and peripheral clinics where only presumptive treatment is available: a randomised controlled trial in Ghana. BMJ 2010, 340:c930. http://dx.doi.org/10.1136/bmj.c930.

9. Witter S, Garshong B: Something old or something new? Social health insurance in Ghana. BMC Int Health Hum Rights 2009, 9:20.

10. National Development Planning Commission: 2008 Citizens' Assessment of the National Health Insurance Scheme of Ghana, Towards a Sustainable Health Care Financing Arrangement that Protects the Poor. Accra: NDPC; 2009.

11. Ministry of Health: Human Resource Policies and Strategies for the Health Sector (2007-2011). Accra: 2007. http://www.moh-ghana.org/UploadFiles/ Publications/hrh_policy_and_plan_08MAY2012.pdf. Accessed 12-07-2014.

12. Turkson PK: Perceived quality of healthcare delivery in rural districts of Ghana. Ghana Med J 2009, 43:2.

13. SEND - Ghana: Balancing Access with Quality Health Care: An Assessment of the NHIS in Ghana (2004-2008). Accra, Ghana: Program Report; 2010.

14. Ministry of Health: Training Manual for the Management of Malaria at Health Facilities in Ghana. Ministry of Health; 2009. http://www.ghanahealthservice. org/includes/upload/publications/TRAINING\%20MANUAL_PART.pdf. Accessed 12-07-2014.

15. Asenso-Okyere WK, Anum A, Osei-Akoto I, Adukonu A: Cost recovery in Ghana: are there any changes in health care seeking behaviour? Health Policy Plan 1998, 13:181-188. http://dx.doi.org/10.1093/heapol/13.2.181.

16. Amexo M, Tolhurst R, Barnish G, Bates I: Malaria misdiagnosis: effects on the poor and vulnerable. Lancet 2004, 364:1896-1898. http://dx.doi.org/ 10.1016/S0140-6736(04)17446-1. 
17. Buabeng KO, Duwiejua M, Dodoo AN, Matowe LK, Enlund H: Self-reported use of anti-malarial drugs and health facility management of malaria in Ghana. Malar J 2007, 6:85. http://dx.doi.org/10.1186/1475-2875-6-85.

18. Whitty CJM, Chandler C, Ansah E, Leslie T, Staedke SG: Deployment of ACT antimalarials for treatment of malaria: challenges and opportunities. Malar J 2008, 7(Suppl 1):S7. http://dx.doi.org/10.1186/1475-2875-7-S1-S7.

19. Donabedian A: Explorations in Quality Assessment and Monitoring, Volume 1: The Definition of Quality and Approaches to its Assessment. Washington: Health Administration Press; 1980.

20. Peterson S, Nsugwa-Sabiti J, Were W, Nsabagasani X, Magumba G, Nambooze J, Mukasa G: Coping with paediatric referral - Ugandan parents' experience. Lancet 2004, 363:1955-1956. http://dx.doi.org/10.1016/50140-6736(04)16411-8.

21. Harteloh PPM: Understanding the quality concept in health care. Accred Qual Assur 2004, 9:92-95. http://dx.doi.org/10.1007/s00769-003-0677-x.

22. Naveh E, Stern Z: How quality improvement programmes can affect general hospital performance. Int I Health Care Qual Assur 1980, 18(4):249-270. http://dx.doi.org/10.1108/09526860510602532s.

23. McLaughlin CP, Kaluzny AD: Continuous Quality Improvement in Health Care. 3rd edition. Sudbury, MA: Jones and Bartlett Publishers; 2006.

24. Ladhari R: A review of twenty years of SERVQUAL research. Int I Health Care Qual Assur 2009, 1(2):172-198. http://dx.doi.org/10.1108/ 17566690910971445.

25. Institute of Medicine: Crossing the Quality Chasm: A New Health System for the 21st Century. Washington, DC: National Academy Press; 2001.

26. Maxwell RJ: Quality assessment in health. Br Med J 1984, 288:1470-1472. http://dx.doi.org/10.1136/bmj.288.6428.1470.

27. Health Services Research Group: Quality of care: 1 . What is quality and how can it be measured? Can Med Assoc J 1980, 146:2153-2158

28. Campbell SM, Roland MO, Buetow SA: Defining Quality of care. Soc Sci Med 2000, 51:1611-1625. http://dx.doi.org/10.1016/S0277-9536(00)00057-5.

29. Winefield HR, Murrell TG, Clifford J: Process and outcomes in general practice consultations: Problems in defining high quality care. Soc Sci Med 1995, 41:969-975. http://dx.doi.org/10.1016/0277-9536(94)00403-G.

30. Peabody J, Taguiwalo M, Robalino D, Frenk J: Improving the quality of care in developing countries. In Disease control priorities in developing world. 2nd edition. Edited by Jamison D. New York: Oxford University Press for the World Bank; 2005:Chapter 70 .

31. Carayon P, Schoofs Hundt A, Karsh B-T, Gurses AP, Alvarado CJ, Smith M, Flatley Brennan P: Work system design for patient safety: the SEIPS model. Qual Saf Health Care 2006, 15(suppl I):i50-i58. doi:10.1136/qshc.2005.015842.

32. Coyle YM, Battles JB: Using antecedents of medical care to develop valid quality of care measures. Int I Qual Health Care 1999, 11(1):5-12. http://dx.doi.org/10.1093/intqhc/11.1.5.

33. Mitchell PH, Ferketich S, Jennings BM: Quality Health Outcomes Model. Health Care: American Academy of Nursing Expert Panel on Quality; 1998:30(1).

34. Donabedian: An Introduction to Quality Assurance in Health Care. New York: Oxford University Press; 2003.

35. Higashi T, Shekelle PG, Adams JL, Kamberg CJ, Roth CP, Solomon DH, Reuben DB, Chiang L, MacLean CH, Chang JT, Young RT, Saliba DM, Wenger NS: Quality of care is associated with survival in vulnerable older patients. Ann Intern Med 2005, 143:274-281. http://dx.doi.org/10.7326/00034819-143-4-200508160-00008

36. Dodoo AN, Fogg C, Asiimwe A, Nartey ET, Kodua A, Tenkorang O, Ofori- Adjei D. Pattern of drug utilization for treatment of uncomplicated malaria in urban Ghana following national treatment policy change to artemisinincombination therapy. Malar J 2009, 8:2. http///dx.doi.org/10.1186/1475-2875-8-2.

37. Kahama-Maro J, D'Acremont V, Mtasiwa D, Genton B, Lengeler C: Low quality of routine microscopy for malaria at different levels of the health system in Dar es Salaam. Malar J 2011, 10:332. http://dx.doi.org/10.1186/1475-2875-10-332.

38. Dalinjong PA, Laar AS: The national health insurance scheme: perceptions and experiences of health care providers and clients in two districts of Ghana. Heal Econ Rev 2012, 2:13. http://dx.doi.org/10.1186/2191-1991-2-13.

doi:10.1186/s12939-014-0063-9

Cite this article as: Fenny et al:: Quality of uncomplicated malaria case management in Ghana among insured and uninsured patients. International Journal for Equity in Health 2014 13:63.

\section{Submit your next manuscript to BioMed Central and take full advantage of:}

- Convenient online submission

- Thorough peer review

- No space constraints or color figure charges

- Immediate publication on acceptance

- Inclusion in PubMed, CAS, Scopus and Google Scholar

- Research which is freely available for redistribution

Submit your manuscript at www.biomedcentral.com/submit
C Biomed Central 